\title{
Display of hepatitis B virus PreS1 peptide on bacteriophage T7 and its potential in gene delivery into HepG2 cells.
}

\begin{abstract}
Hepatitis B is a major public health problem worldwide which may lead to chronic liver diseases, cirrhosis and hepatocellular carcinoma. An interaction between hepatitis B virus (HBV) envelope protein, particularly the PreS1 region, and a specific cell surface receptor is believed to be the initial step of HBV infection through attachment to hepatocytes. In order to develop a gene delivery system, bacteriophage T7 was modified genetically to display polypeptides of the PreS1 region. A recombinant T7 phage displaying amino acids 60-108 of the PreS1 region (PreS1(60-108)) was demonstrated to be most effective in transfecting HepG2 cells in a dose- and time-dependant manner. The phage genome was recovered from the cell lysate and confirmed by PCR whereas the infectious form of the internalized phage was measured by a plaque-forming assay. The internalized phage exhibited the appearance of green fluorescent dots when examined by immunofluorescence microscopy. Surface modification, particularly by displaying the PreS1(60-108) enhanced phage uptake, resulting in more efficient in vitro gene transfer. The ability of the recombinant phage to transfect HepG2 cells demonstrates the potential of the phage display system as a gene therapy for liver cancer.
\end{abstract}

Keyword: Hepatocytes; Transduction; Hepatitis B surface antigen; Phage vectors. 